\title{
Comparative Transcriptomic and Metagenomic Analysis Reveal the Key Factors on the Growth Rate in Red Swamp Crayfish (Procambarus Clarki)
}

Duanduan Chen

Shandong Agricultural University

Leifeng Guo

Shandong Agricultural University

Hui Wang ( $\sim$ wanghui2328@sdau.edu.cn )

Shandong Agricultural University

Research article

Keywords: Procambarus clarkii, genetic background, hepatopancreas transcriptome, intestinal microbial structure

Posted Date: June 24th, 2021

DOI: https://doi.org/10.21203/rs.3.rs-645967/v1

License: () (i) This work is licensed under a Creative Commons Attribution 4.0 International License. Read Full License 


\section{Abstract}

Background: Although red swamp crayfish (Procambarus clarkii) is one of the most important species in aquaculture, the factors that influence differences in growth and development between individual siblings are still not fully understood. To address this lack of knowledge, we designed experiments to elucidate factors contributing to individual differences by comparing the hepatopancreatic transcriptome and gut flora structure of individuals with differences in sibling crayfish size under the same rearing conditions and attempted to find links between gut flora and host transcriptome information.

Result: In total, 300691028 high-quality reads were obtained that were used to assemble 60637958 unigenes. Comparison of the expression profiles of the hepatopancreas of crayfish of different sizes revealed 497 differentially expressed genes $(P<0.05)$. A total of 32 KEGG signaling pathways were found to be enriched after the KEGG database and GO functional annotation analyses, with the highest number of unigene enrichments related to organismal metabolism. Additionally, we found that proteobacteria, tenericutes, actinobacteria, and bacteroidetes made up a larger portion of the microbiome of larger individuals, suggesting that crayfish microbiota adapts to rapid growth, which may promote accelerated development by regulating the expression level of relevant genes.

Conclusions: This work has accumulated data to support the potential impact of structural alterations in gut flora under equivalent feeding conditions in explaining the differential expression of transcriptomic genes among differentially developing individuals of sibling crayfish. These results further elucidate how the intestinal environment affects the rapid development of invertebrate crustaceans and provide a reference for further understanding of the regulatory mechanisms of the host's in vivo environment.

\section{Highlight}

\section{Same genetic background}

2. The differential development of siblings

3. Transcriptomic and microbial structure analysis in two sequencing approaches.

4. Analysis of potential factors affecting growth velocity

\section{Background}

The red swamp crayfish (Procambarus clarkii) is native to the south-central United States (primarily in Louisiana) and northeastern Mexico, but has been transplanted around the world [1-3]. P. clarkii is a freshwater crayfish, which is economically significant due to its culinary uses [4, 5]. Additionally, it is omnivorous and has a rapid growth rate and high environmental adaptability [6]. In recent years, $P$. clarkii has become an important economic farming species in China.

The intestinal tract is the most important digestive and absorption organ of crustaceans, including shrimps and crabs, and hosts a large number of complex microorganisms that are interdependent and mutually constrained by their hosts, forming a unique intestinal microecosystem [7]. The relationship between gut microbes and their hosts has been shown to play a crucial role in growth rate and disease susceptibility in several different species, due to its impact on nutrient processing, energy balance, as well as growth and development in the host [8]. Shrimp and crayfish lack an acquired immune system and rely on various types of non-specific immune factors to recognize foreign objects and pathogens [9]. The structure and function of the normal flora in the intestine of shrimp and fish has been shown to have a significant impact on their immune systems due to its ability to form a powerful barrier against pathogenic bacteria [10-12].

The hepatopancreas is an important multifunctional organ of crustaceans, which plays a role in metabolism, nutrient absorption, and immune function [13]. Studies have also found that changes in the hepatopancreas of crayfish can be caused by the accumulation of heavy metals and affect the ability of species to cope with various environmental stresses [14]. Recent studies have sequenced and analyzed the transcriptomes of ocular stalk, hepatopancreas, muscle, gills, intestine, blood cells, and stomach of crayfish in order to better understand environmental adaptation and response to disease, but there is a lack of transcriptomic data involving the role of intestinal flora in growth and development among siblings $[15,16]$. To fill this current knowledge gap, this study employed transcriptomic data to analyze the role that the gut flora and the host transcriptome play in affecting the growth rate of $P$. clarkii. These studies further elucidate how the intestinal environment affects the rapid development of invertebrate crustaceans, providing insights to further understand the regulatory mechanisms of the host's internal environment and to understand the safety of the host's internal environmental homeostasis.

\section{Results}

\section{Transcriptome sequencing and assembly}

Hepatopancreatic mRNA from differentially developing sibling individuals was detected by RNA-Seq, with three replicates per group. Members of the smaller group were termed SI1, SI2, and SI3, while members of the larger group were termed LI1, LI2, and LI3. After the removal of low-quality reads, clean reads were assembled into reference sequences for subsequent analyses using Trinity [18]. The LI group sequencing reads produced 158810616 unigenes, while the SI group yielded 141880412 unigenes. The error rate of the samples was restricted between 0.02 and 0.03 , with Q20 accounting for more than $97 \%$ (Table 1). The reference sequence set included 135061303 transcripts and 60637958 unigenes, with unigene (gene) N50 values of 2468 and N90 values of 511 in the samples, respectively (Table 2).

After the spliced transcripts were obtained, they were functionally annotated (Table 3). The proportion of all genes annotated to the database were $\mathrm{Nr} 14505$ (32.68\%), Nt 567 (12.79\%), swiss-prot 5684 (12.8\%), Pfam 10306 (23.22\%), G0 14373 (32.39\%), and KOG 5889 (13.27\%). The annotation results identified 44372 genes. Of these, 2090 Unigene were matched to the five databases (Fig. 1) 


\section{Identification of DEGs}

After quantification of gene expression levels, differential expression analysis was performed using screening criteria (| log2 (fold change) |> 1 and adjusted pvalue $<0.05$ ) to identify genes with significant differences between different samples. In brief, 497 DEGs were found in the LI vs SI group, of which 221 were up-regulated and 276 were down-regulated (Fig 2A). The analysis of the significance of the DEGs is shown in Figure 2B. Significantly DEGs were plotted into clustered heat maps based on FPKM (expected number of Fragments Per Kilobase of transcript sequence per Millions base pairs sequenced)) values, as shown in Figure 3.

The annotated genes were then categorized into five branches according to KEGG metabolic pathways (level 2): Cellular Processes, Environmental Information Processing, Genetic Information Processing, Genomic Information Processing, Metabolism and Organic systems. In cellular processes, there were 398 unigenes in transport and catabolism and 229 unigenes in cell community signaling. In environmental information processing, the number of unigenes in signal transduction (601) was greater than the number in signaling molecules and interacting transport. The number of unigenes annotated in translation, folding, sorting, and degradation based on information processing was 320 , and the number of unigenes in lipid metabolism and carbohydrate metabolism was approximately 250 . Among the five branches of KEGG, the metabolic pathways were the most numerous. Among the immune signaling pathways, the endocrine system (368) and the immune system (251) were found in the most and second most unigenes, respectively. The organic system ranked second among the five branches. Both metabolic and organic systems were closely related to organismal growth (Fig. 4).

\section{Validation of RNA-Seq with RT-qPCR}

To further validate the accuracy of our RNA-Seq data, we selected 13 genes for RT-qPCR (Tables S1 and S2). These genes included members involved in apoptosis, immunity and organismal metabolism. Figure 5 shows that all RNA sequences are consistent with the RT-qPCR results. Therefore, the RNA-Seq data were considered to be accurate and reliable in this study. The RT-qPCR results also showed that there were significant differences in the expression of genes associated with metabolism and immunity across different individuals.

\section{Taxonomic composition of intestine microbiota}

At the end of the feeding cycle, high-throughput sequence analysis of bacterial 16S ribosomal RNA v3-v4 region was performed on the intestinal contents of $P$. clarkii. As shown in Supplemental Table S3, the top four most abundant bacteria in the LI group were Proteobacteria (49.22\%), Tenericutes (4.66\%), Actinobacteria (3.11\%), and Bacteroidetes (2.97\%). In the SI group, Proteobacteria, Firmicutes, Actinobacteria, and Chloroflexi were the four most abundant phyla, accounting for $39.57 \%, 5.83 \%, 5.35 \%$, and $5.34 \%$, respectively.

Based on the sequence information available for each sample, clustering was performed at both the species and sample levels to create heat maps (Fig $6 \mathrm{~A}$ ). The main genera in the LI group were Novosphingobium, Legionella, Acinetobacter, Bosea, Microbacterium, Flavobacterium, Rhodobacter, Pseudorhodobacter, and Gemmobacter. The major bacterial genera in the SI group were Vogesella, Bacillus, Aeromonas, Bacteroides, Defluviicoccus, Acinetobacter, Gemmobacter, Rhodobacter, Cupriavidus, and Gaiella (Fig 6). Interestingly, Gemmobacter was was highly abundant in both groups.

To further investigate the phylogenetic relationships among bacterial genera, representative sequences of the top 100 genera were compared by multiple sequence alignment (Fig. 7). The species richness of Vogesella, Bacillus, Aeromonas, Bacteroides, Defluviicoccus, Acinetobacter, Gemmobacter, and Rhodobacter were further compared at the genus level. The results showed significant differences $(\mathrm{P}<0.05)$ in $\mathrm{LI}$ vs SI in Gemmobacter, Acinetobacter, Candidatus-Bacilloplasma, Vogesella, Rhodobacter, Paracoccus, and Bosea.

\section{Microbial Function Prediction and Functional Enrichment of the Hepatopancreas Transcriptome}

Annotation results of gut microbial function showed that crayfish appeared to have a higher proportion of KEGG enrichment in the environmental information and metabolism categories (Fig. 8A). In the metabolism category, carbohydrate metabolism and amino acid metabolism were the most significantly enriched categories. Membrane transport was found to be most abundant in the environmental information processing category.

A scatterplot was used to generate a graphical representation of the KEGG enrichment of the transcriptome DEGs from previous analyses, with the 20 most enriched pathway entries displayed (Fig. 8B). This list included fatty acid biosynthesis, phototransduction, prion diseases, riboflavin metabolism, fructose, mannose metabolism, ether lipid metabolism, tyrosine metabolism, propanoate metabolism, PPAR signaling pathway, glycolysis/gluconeogenesis, cysteine and methionine metabolism, amyotrophic lateral sclerosis (ALS), drug metabolism, adipocytokine signaling pathway, melanogenesis, citrate cycle (TCA cycle), AMPK signaling pathway, vascular smooth muscle contraction, insulin signaling pathway, and lysosome. The majority of these terms were associated with nutritional metabolism or immune processes FigureS1.

\section{Intestinal biopsy analysis and humoral factor testing}

The results of the humoral immune factor assay showed that the activity of SOD was significantly higher in the LI group than in the SI group ( $<<0.05$ ), and the activity of LI Ppo and Lyz were higher than in the SI group (Fig 9A). GIs, AMS, and lipase in body fluids of the LI group was significantly more active than those of the SI group $(\mathrm{P}<0.05)$ (Fig 9B).

The developmental differences in the intestinal tissue sections of the P. clarkii (Fig 10) revealed that the mucosal folds of the LI group were intact (Fig10A), with a higher height, tighter arrangement, larger folds, and epithelial cells in the mucosal folds arranged in an orderly manner with smooth striated edges on the surface (Fig10B); the intestinal villi of the SI group were sparsely arranged and short, and the depth of the intestinal crypt was deepened.

\section{Discussion}


In this study, we used RNA-Seq and 16S ribosomal RNA-Seq to analyze the hepatopancreatic transcriptome and the intestinal microflora structure of $P$. clarkii, to identify factors that affect the growth and development of the crayfish (Fig S2). Our findings provided a basis for better understanding of the biology of this species at the molecular level and the intestinal microflora level. In this study, RNA-Seq and bioinformatics analyses were used to study the dry pancreas transcriptome information of different individuals with the same genetic background and the same feeding conditions. There were significant differences in the expression of 497 genes between large and small groups (276 up-regulated and 221 down-regulated genes). The GO annotation and KEGG pathway analyses showed that the DEGs were enriched in catalytic activity and binding of metabolic processes in the biological processes category, and single-cell, cellular processes, and cellular components of the cellular and membrane in the molecular functions category. Analysis of the transcriptomics data also revealed many key genes and important pathways related to immune and metabolic processes (Figs. 1 and 2).

We mainly validated genes that were up-regulated with RT-qPCR(Fig 5), including FASN (fatty acid synthase, animal type), SOD1 (superoxide dismutase, cu-zn family), LSC2 (succinyl-coa synthetase beta subunit), TYR (tyrosinase), CALM (calmodulin), ACC1 (acetyl-coa carboxylase/biotin carboxylase 1), ADIPOR (adiponectin receptor), CTSL (cathepsin L), ACLy (ATP citrate (pro-s)-lyase), and FATP1 (fatty acid transporter). These genes were primarily involved in biological processes associated with fatty acid biosynthesis, peroxisome, lysosome, riboflavin metabolism, estrogen signaling pathway, AMPK signaling pathway, antigen processing and presentation, fatty acid degradation, and the PPAR signaling pathway. FASN is an important enzyme in lipid metabolism and plays a role in a variety of biological processes. In mammals, FASN is associated with immunity and anti-bacterial activity [19]. Fatty acids can regulate phagocytic activity, the production of inflammatory cytokines and immunoglobulin, as well as proliferation, apoptosis, and migration of immune cells. It has been reported that palmitate, a product of FASN mediated biosynthesis, can promote monocyte adhesion by activating various immune factors in endothelial cells of human adipose tissue in the microvascular system $[20,21]$. Recent studies have found that when crayfish are infected with White Spot Syndrome Virus (WSSV), FASN expression can be induced through the PI3K/AKT pathway, improving lipid biosynthesis to support the morphogenesis of the virus, which suggests that FASN may play a positive role in WSSV infection (DOI:10.1016/j.dci.2015.06.0010). In order to gain insight into the key role of the FASN gene in the metabolic process, we investigated the specific involvement of FASN in the Fatty acid biosynthesis signaling pathway, which shows that FASN is involved in almost all aspects of the process. Investigating the specific regulatory mechanisms of the FASN gene in the growth process will be the focus of our next study. The FATP family is a group of proteins that is predicted to be part of a fatty acid transport pathway. Six distinct subtypes of FATP have been identified in mammalian systems, which take part in the importation of exogenous fatty acids or the activation of long-chain fatty acids. It remains controversial whether these proteins function as membrane-bound fatty acid transporters or as acyl-coa synthases, which activate long-chain fatty acids and accompany their transportation [22]. The damage of aging on the body is usually related to certain structural and functional defects in organs [23]. These defects include oxidative modification, protein aggregation, and differential expression of specific genes. Studies in mammals have found that certain oxidative damage is related to aging (DOI: 10.1016/j.exger.2019.110795). SOD1 is an important member of the super-oxidase family, which has been demonstrated to have ROS scavenging ability [24]. During growth, the body produces a large amount of non-specific oxidation molecules such as proteins, nucleic acids, and lipids, and SOD1 acts as an oxidative scavenger to effectively control the active oxygen content, thereby reducing the accumulation of oxidative damage. Recent studies have found that SOD1 can also take part in metabolism, transcription, and maintenance of redox balance [25]. Our differential expression analysis showed that SOD1 gene expression was significantly higher in the LI group than in the SI group. Tyrosinase can be found in animals, plants, and fungi, and has a broad spectrum of substrates for a variety of phenolic compounds (L-tyrosine, L-DOPA, catechol, caffeic acid, tyramine, phenol, p-aminophenol, cresol, p-cresol, dopamine, 4-hydroxyanisole, L-isoproterenol, 4-ethoxyphenpl, 4-butylcatechol, and pyrogallol) [26]. Overall, these DEGs in the hepatopancreas may explain differential development of different $P$. clarkii, individuals with the same genetic background.

A healthy microbiome is not a constellation of individual species, but rather includes a network of interdependent microbes [27]. The microbiota in the gastrointestinal tract is a symbiotic partner of the host and is crucial for intestinal microvasculature development, metabolism and the development of multiple components of the host immune system[28,29]. The vertebrate intestine hosts $10^{14}$ bacterial cells, including perhaps tens of thousands of bacterial strains [27]. Previous work in Litopenaeus vannamei demonstrated the close relationship between intestinal microbes and intestinal development, with beneficial intestinal flora contributing to overall host health [30]. In addition, intestinal flora can also have a protective effect against pathogens and can stimulate the host immune system through cell signaling in addition to producing antibacterial peptides and competing for adhesion locations (DOI:10.1038/nature11552). Improving the absorption of nutrients during the farming of aquatic animals is one key to increasing yield and quality. Some species, such as Penaeus vannamei, have been reported to select microorganisms for specific functions according to their needs under certain selective pressures [31].

We found that at the phylum level, Proteobacteria were the most abundant intestinal bacteria in both groups. Proteobacteria has the largest phylogenetic composition and is comprised of 116 validated families. On average, there are 10.1 families per validated phylum (median, 3.0), according to the List of Prokaryotic Names with Standing in Nomenclature (LPSN). Members of the phylum Proteobacteria have greatly variable morphology and diverse physiology, which makes them competitive in surviving in various ecological niches [32]. Proteobacteria have been observed to be ubiquitous in habitats such as soil [32], plants [33], and seawater[34]. Most microorganisms in the animal gut are obligate anaerobes, but Proteus is an interstitial anaerobic bacterium that we speculate may affect host oxygen homeostasis. Microbiomes that are rich in diversity can provide the host with complementary genetic resources, which have an effect on energy extraction, essential vitamin production, intestinal maturation, and immune system development. Furthermore, many pathogenic Vibrio species in this family are potentially harmful to crawfish [35]. In the larger individual group, the proportion of Actinobacteria was second only to Proteobacteria (Table S3). Studies have shown that the larva development of South American white shrimp can alter the structure and function of intestinal bacteria, with the proportion of Actinobacteria increasing at a certain stage of development [36].

In this study, the proportions of Firmicutes and Actinobacteria bacteria in the SI group were relatively high. This correlation suggests that gut bacteria may influence the speed at which the body develops over the same time. The species richness of Vogesella, Bacillus, Aeromonas, Bacteroides, Defluviicoccus, Acinetobacter, Gemmobacter, and Rhodobacter was further compared at the genus level (Fig. 7). Gemmobacter, Acinetobacter, Candidatus-Bacilloplasma, Vogesella, Rhodobacter, Paracoccus, and Bosea had a significantly different richness in the LI group compared to the SI group ( $\mathrm{p}<0.05$ ). 
The genus Vogesella belongs to the class Betaproteo and includes three recognized heterotrophic species (Fig. 7), including V. indigofera ATCC 19706T, Pergesida perlucida LMG 24214T and V. lacus LMG 24504T [37], which were all isolated from freshwater sites (in sediment or water). Some studies have confirmed the ability of these genera to degrade peptides and several other polymers. This is contrary to the current understanding that members of the genus Vogesella mainly degrade low molecular weight organic compounds, although this genus has not been studied extensively [38]. The different proportion of the taxonomic abundance of Rhodobacter represents an interesting finding since members of this genus can inhibit the growth of Vibrio[39]. Therefore, bacteria of this family are widely used as probiotics and be very efficacious[39]. According to some reports, although there are some Acinetobacter species in the microbiome of healthy human skin and animal intestines, many isolates recovered from clinical specimens show substantial pathogenicity [40]. Studies have shown that the secondary metabolites provided by the gut microbiota take part in the biologically active functions required by the host [41]. Thus, regulation of the host microflora may represent a way to manage the growth of healthy host species.

By comparing the intestinal microbiota of the LI and SI groups, we found differences in many species associated with responding to environmental stress or preventing infection (Fig. 8). Carbohydrate metabolism function prediction at the second KEGG level revealed enrichment in pathways associated with transport, information-based processing, cellular processes, and signaling. Xenobiotics biodegradation and metabolism, carbohydrate metabolism, transport, genetic information processing, cellular-processes-and-signaling, and transcription processes are critical pathways for crayfish growth. Studies have shown that secondary metabolites provided by the gut flora are involved in essential biological functions of the host and analysis of transcriptomic KEGG data revealed that many genes are involved in the metabolic and immune processes of the organism. Exploring the intrinsic connection between the two sequencing approaches that we employed in the future will undoubtedly yield new findings related to the relationship between $P$. clarkii microbiome and growth rate.

The intestinal tract is often subject to microbial parasitism, which requires pathogenic bacteria to adhere to the intestinal mucosa. The genetic diversity of different species of crayfish, despite their similar kinship, is still present, and the differences in transcription and translation of structural and functional genes have implications for growth and development. The physicochemical properties of the intestinal tract and the type of nutrients also determine the structure and function of the adherent symbiotic intestinal microorganisms.

The rate of development of crayfish is influenced by many factors, including host and non-host factors. The host factors are genetic, developmental, and physiological state specific, which in turn affect the specificity of the gut flora. The non-host factors (water environment, feed, drugs, and probiotics) may also affect gut microorganisms in the acquired environment. Overall, the complex and variable gut flora is a balanced system of interactions between host, environment, and microorganisms.

\section{Conclusion}

In this study, we analyzed the differential expression of the hepatopancreatic transcriptome and compared differences in intestinal microflora structure between small and large crayfish siblings reared under the same conditions. We found differences in gene expression related to growth and development among different offspring, despite similar genetics. The rapidly growing individuals showed significant differences in the expression of genes that are involved in coping with environmental changes, dealing with disease infection, and the processing of metabolism related to growth. The structure of the microbiome may also play a role in these processes since the structure of intestinal microflora adapts to the environment to promote the development of an organism. In addition, transcriptomic and $16 \mathrm{~S}$ ribosomal RNA results, including significantly enriched GO and KEGG pathways and DEGs involved in organism development, provide further evidence that differences between individuals with different growth rates are accompanied by changes in intestinal flora structure. Our findings have implications for understanding the roles that the host and non-host environments play in influencing growth differences among crayfish individuals.

\section{Materials And Methods}

\section{Selection and feeding of experimental animals}

P. clarkii were selected from sexually mature individuals from the Dongping Lake area of Tai'an, Shandong Province, China. Specimens (35-40 g) of male and female crayfish were cultured in circulating water at $24-27^{\circ} \mathrm{C}$. Feed was purchased from New Hope Liuhe Group (Linyi, China). After stabilizing the samples for one week, one male and four females were selected from healthy individuals and reared in the same tank (2 $\mathrm{m}$ diameter and $30-40 \mathrm{~cm}$ depth) for mating. After mating, the fertilized female crayfish were reared individually until the juvenile crayfish hatched successfully. All juveniles $₫ 280$ in total, were fed under the same conditions until 45 days of age, during which feed, water temperature, density, dissolved oxygen, nitrite, and ammonia levels were kept within the appropriate ranges.

\section{Collection of intestinal contents and hepatopancreas}

Animals were selected and collected after 45 days of feeding. Six of the largest and smallest healthy individuals were selected to represent the large and small study groups. The crayfish were sterilized with $75 \%$ alcohol, followed by extraction of as much blood as possible from the pericardial cavity with a $1 \mathrm{ml}$ syringe with anticoagulant added inside the syringe in advance until after the experimental animals were incapacitated, followed by dissection of the hepatopancreas and intestinal contents, which were then aseptically placed in a $1.5 \mathrm{ml}$ centrifuge tube and frozen in liquid nitrogen before storage at $-80^{\circ} \mathrm{C}$, and hemolymph was stored in $1.5 \mathrm{ml}$ Eppendorf tubes, subjected to $10 \mathrm{~min}$ of centrifugation at $4000 \mathrm{rpm}$ at $4^{\circ} \mathrm{C}$. The supernatant (serum) was collected, packaged, and stored at $-80^{\circ} \mathrm{C}$. The intestines of experimental animals from the $\mathrm{LI}$ and $\mathrm{SI}$ groups were collected and analyzed for tissue sections.

RNA extractions, library construction and RNA sequencing 
RNA was isolated using Trizol Reagent, according to the manufacturer's instructions (Accurate Biotechnology Co. Ltd, Hunan, China). The quality and integrity of these total RNA samples were determined by a NanoDrop 2000 spectrophotometer (NanoDrop Technologies, Wilmington, DE, USA). 1.5\% agarose gels were used to determine RNA integrity. We constructed sequencing libraries from gills using a TruseqTM RNA sample prep Kit for the Illumina HiSeq 2000 platform, following the manufacturer's instructions.

\section{Transcriptome assembly and functional annotation of unigenes}

A total of $1.5 \mu \mathrm{g}$ RNA per sample was used as input material for RNA sample preparation. Sequencing libraries were generated using the NEBNext ${ }^{\circledR}$ Ultra ${ }^{\mathrm{TM}}$ RNA Library Prep Kit for Illumina ${ }^{\circledR}$ (NEB, USA), following the manufacturer's recommendations. Samples were then barcoded, followed by clustering and sequencing using Illumina sequencing technology (Novozymes Biotechnology, Beijing, China). In this step, reads containing adapters, reads containing ploy-N, and low-quality reads were removed from the raw data to obtain clean reads. The Q20, Q30, GC-content, and sequence repeatability of the clean data were also calculated.

\section{Gene functional annotation}

All downstream analyses were based on high-quality clean reads. BLAST was used to compare unigene sequences against the NCBI non-redundant protein (Nr) (http://www.ncbi.nlm.nih.gov/), NCBI nucleotide sequences (Nt) (https://www.ncbi.nlm.nih.gov), Protein family (Pfam) (http://pfam.sanger.ac.uk/), Gene Ontology (GO) (http://www.geneontology.org/), Clusters of Orthologous Groups of proteins KOG: euKaryotic Ortholog Groups (KOG/COG) (http://www.ncbi.nlm.nih.gov/COG/), eggNOG (database of orthologous groups of genes) (http://eggnogdb.embl.de/), and Kyoto Encyclopedia of Genes and Genomes (KEGG) (http://www.genome.jp/kegg/) databases. KOBAS 2.0 was used to obtain KEGG orthology results for unigenes in KEGG.

\section{Analysis of differentially expressed genes (DEGs) and functional enrichment}

Differential expression analysis of two conditions/groups was performed using the DESeq R package (1.10.1). DESeq provide statistical routines for determining differential expression in digital gene expression data using a model based on the negative binomial distribution. The resulting $P$ values were adjusted using the Benjamini and Hochberg's approach for controlling the false discovery rate. Genes with an adjusted P-value $<0.05$ found by DESeq were assigned as differentially expressed

DESeq was utilized to identify DEGs across samples which contained biological replicates. The resulting DEGs were then subjected to enrichment analysis of the three major categories of GO (biological process [BP], cellular component [CC], and molecular function [MF]). Next, KEGG analysis was carried out to identify significantly enriched metabolic pathways using KOBAS (2.0).

\section{Validation by quantitative Real-Time PCR (RT-qPCR).}

RT-qPCR was used to validate selected candidates from the DEGs which were previously identified using RNA-Seq. RT-qPCR reactions were carried out using a LightCycler ${ }^{\circledR} 2.0$ (Roche). A partial sequence of the P. clarkii 18S ribosomal RNA gene (NCBI:AF436001) was used as the reference gene. The PCR was performed using a SYBR Premix Ex Taq kit (Accurate Biotechnology Co., Ltd), and the $20 \mu$ I PCR reaction systems consisted of $10 \mu$ l SYBR Premix Ex Taq ( $2 \times$ ), $0.4 \mu \mathrm{l}$ of each gene-specific primer $(10 \mathrm{nmol}), 2 \mu \mathrm{l}$ cDNA, and RNase free water up to $20 \mu \mathrm{l}$. Cycling conditions were $95^{\circ} \mathrm{C}$ for $30 \mathrm{~s}$, followed by $40 \mathrm{cycles}$ of $95^{\circ} \mathrm{C}$ for $5 \mathrm{~s}$ and $60^{\circ} \mathrm{C}$ for $30 \mathrm{~s}$. Each sample was run in triplicate.

\section{Sequencing and data processing of intestinal microbial genomic DNA}

Total genomic DNA from samples was extracted using the CTAB/SDS method. DNA concentration and purity were monitored on $1 \%$ agarose gels. According to the concentration, DNA was diluted to $1 \mathrm{ng} / \mu \mathrm{l}$ using sterile water. All PCR reactions were carried out in $30 \mu \mathrm{l}$ reactions with $15 \mu \mathrm{l}$ of Phusion ${ }^{\circledR}$ High-Fidelity PCR Master Mix (New England Biolabs), $0.2 \mu \mathrm{M}$ of forward and reverse primers and approximately $10 \mathrm{ng}$ template DNA. Thermal cycling consisted of initial denaturation at $98^{\circ} \mathrm{C}$ for $1 \mathrm{~min}$, followed by 30 cycles of denaturation at $98^{\circ} \mathrm{C}$ for $10 \mathrm{~s}$, annealing at $50^{\circ} \mathrm{C}$ for $30 \mathrm{~s}$, and elongation at $72^{\circ} \mathrm{C}$ for $30 \mathrm{~s}$. Reactions were finished with a final $5 \mathrm{~min}$ at $72^{\circ} \mathrm{C}$. Sequencing libraries were generated using an lon Plus Fragment Library Kit (Thermo Scientific), following manufacturer's recommendations. The library quality was assessed using a Qubit 2.0 Fluorometer (Thermo Scientific). Finally, the library was sequenced on an lon S5TM XL platform to generate $400 / 600$ bp single-end reads.

To study the species composition of each sample, operational taxonomic unit (OTU) clustering was performed on valid tags with $97 \%$ identity for all samples, and then annotated with representative sequences of OTUs. Cutadapt (V1.9.1, http://cutadapt.readthedocs.io/en/stable/) was used to prune low-quality reads. After initial quality control, chimeric sequences were removed by comparison with the species annotation database. The Read Sequence (https://github.com/torognes/vsearch/) Upanish v7.0.1001 software (http://www.drive5.com/uparse/) was employed for clustering clean reads from all samples. Sequences with $97 \%$ identity were clustered, and the sequence with the highest OTU frequency was selected as the representative sequence. OTU sequences were annotated using the Mothur method, and species annotation analysis was performed using the SSUrRNA database of SILVA 132 (http://www.arb-silva.de/), with thresholds set at 0.8 to 1.0. Classification information and statistics were generated for each taxonomic level, including kingdom, boundary, order, family, genus, and species.

\section{Intestinal microbial function prediction methods}

Functional prediction was carried out using the nearest neighbor method based on the similarity of 16S ribosomal RNA sequences in the R package Tax4Fun [17]. Specifically, 16S ribosomal RNA sequences from all prokaryote genomes were extracted from the KEGG database and then were compared to the SILVA, SSU Ref, and Nr databases (BLAST bit score >1500) using the BLASTN algorithm to establish a correlation matrix. The KEGG database results, annotated via UProC and PAUDA, were mapped to the SILVA database for functional annotation. 


\section{Histological analysis}

The intestines of experimental animals in the LI and SI groups were collected and analyzed in tissue sections. After paraformaldehyde fixation for $24 \mathrm{~h}$, the intestines were embedded in paraffin blocks and dehydrated with different concentrations of ethanol. The blocks were sectioned to $5 \mu \mathrm{m}$ thickness with a slicer, stained with hematoxylin and eosin, and resin-fixed for examination by an optical microscope.

\section{Humoral factor testing}

To detect the viability of humoral factors in animals at different group, hemolymph supernatants from each group as described in 2.1 were randomly selected and mixed according to group distribution, with three biological replicates for each group. The activities of plasma phenol oxidase (Ppo), lysozyme (Lyz), glutaminase (GIs), lipase, amylase (AMS), and superoxide dismutase (SOD) of the aforementioned humoral factors were determined using a commercial assay kit (Nanjing Jiancheng Institute, Nanjing, China) according to the manufacturer's instructions.

\section{Statistical analysis}

Statistical analysis was performed using SPSS Version 21.0 (IBM Corporation, USA) and GraphPad Prism version 8.0 (GraphPad Software Inc., San Diego, CA, USA) software. Statistical differences were determined via one-way analysis of variance (ANOVA) and Tukey's multiple range tests. Significance and high significance were set at $p$-value $<0.05$ and $p$-value $<0.01$, respectively.

\section{Abbreviations}

Not applicable

\section{Declarations}

\section{Ethics approval and consent to participate:}

This study was conducted following the 2012 International Guidelines for Biomedical Research Involving Animals (Council for International Organizations of Medical Sciences, http://www.cioms.ch), the care and use of laboratory animals were in full compliance with these guidelines. Furthermore, the study species is not listed in the List of Protected Animals in China and experimental research on this species is legal in China囚no institutional permission was required for the collection of animals in this study.

\section{Consent for publication}

Not applicable.

\section{Availability of data and materials section:}

The datasets generated and/or analyzed during this study are not publicly available due to copyright and the progress of current research, and we will also delve into specific mechanisms and applications, but are available from the corresponding authors upon reasonable request.

\section{Competing interests}

The authors declare that they have no competing interests.

\section{Funding:}

This work was financially supported by,1): The engineering project of the Agricultural Fine Breeding of Shandong Province (breeding for good quality and high resistance breakthrough new varieties of penaeid, No. 2019LZGC014);2): The key project of the Agricultural Fine Breeding of Shandong Province (No. 2017LZN010);3):Science and technology demonstration project of "Bohai granary" in Shandong Province (Innovation of ecological symbiotic agriculture model and integrated demonstration of efficiency enhancement technology in saline alkali wetland, No. 2019BHLC006);4):Agricultural Science and Technology Fund of Shandong Province (Research and demonstration of poverty alleviation in science and technology industry based on the construction of innovation bases for offspring seed breeding in Dongping (Yellow River Flood Plains), No. 2019YQ032);5):Modern Agricultural Industry Technical System of Shandong Province (SDAIT-13-02);6):Postgraduate education innovation projects of Shandong Province (SDYY11083; SDYC12048);7):Agricultural expert advisory group of Shandong Province;8):The "Double First-Class Discipline" Construction Foundation of Shandong Province. The funders had no role in the design of the study and collection, analysis, and interpretation of data and in writing the manuscript.

\section{Authors' contributions:}

Affiliations:

Aquaculture Research Lab, College of Animal Science and Veterinary Medicine, Shandong Agricultural University. Duanduan Chen, Leifeng Guo, Hui Wang.

\section{Contributions:}

DC: Data curation, Writing - original draft, Writing - reviewing \& editing, Conceptualization, Methodology, Software. 
LG: Visualization, Investigation.

HW: Writing - reviewing \& editing.

All authors have read and approved the manuscript.

\section{Acknowledgments:}

The authors thank Shandong Agricultural University for providing the experimental infrastructure and the Dongping breeding base in Shandong for providing the breeding facilities, and we thank the supporters of the various grants, and we would like to thank the editors of the BMC journal for providing helpful and constructive comments that improved the manuscript substantially.

\section{References}

[1] J.V. Huner, J.E. Barr, E.B. Coleman, Red Swamp Crawfish: Biology and Exploitation, (1984).

[2] R., Jiménez, X., Romero, Iron deposits on the cuticle and in the hepatopancreas of the Australian redclaw crayfish, $<$ em $>$ Cherax quadricarinatus $<$ /em $>$ (Von Martens), Journal of Fish Diseases (1998).

[3] C. Manfrin, M. Tom, G. De Moro, M. Gerdol, P.G. Giulianini, A. Pallavicini, The eyestalk transcriptome of red swamp crayfish Procambarus clarkii, Gene 557(1) (2015) 28-34.

[4] Nan, Jiang, Xiaoyi, Pan, Zemao, Gu, Wenzhi, Liu, Kaige, Si, Proliferation dynamics of WSSV in crayfish, Procambarus clarkii, and the host responses at different temperatures, Journal of fish diseases (2019).

[5] M.L.A. B, L.L. A, M.N.A. C, S.K. C, J.W.Z. D, Z.Z.Y. A, X.Y.Q. A, X.M.Z. E, S.H.C. A, L.S.D. A, Involvement of gamma interferon inducible lysosomal thiol reductase in the innate immune responses of red swamp crayfish, Procambarus clarkii, Developmental \& Comparative Immunology 99.

[6] H. Zhang, X. Yao, Y. Ding, Z. Xu, R. Liang, Y. Zhang, Y. Wu, B. Li, B. Guan, PI3K signaling pathways modulated white spot syndrome virus (WSSV) replication in Procambarus clarkii, Fish \& Shellfish Immunology (2018) S1050464818301074.

[7] J. Cahenzli, Y. Köller, M. Wyss, M.B. Geuking, K.D. McCoy, Intestinal microbial diversity during early-life colonization shapes long-term IgE levels, Cell Host Microbe 14(5) (2013) 559-70.

[8] A. Abid, S.J. Davies, P. Waines, M. Emery, M. Castex, G. Gioacchini, O. Carnevali, R. Bickerdike, J. Romero, D.L. Merrifield, Dietary synbiotic application modulates Atlantic salmon (Salmo salar) intestinal microbial communities and intestinal immunity, Fish \& Shellfish Immunology 35(6) (2013) $1948-1956$.

[9] T. Sangkuanun, S. Wichienchot, Y. Kato, H. Watanabe, S. Peerakietkhajorn, Oligosaccharides derived from dragon fruit modulate gut microbiota, reduce oxidative stress and stimulate toll-pathway related gene expression in freshwater crustacean Daphnia magna, Fish Shellfish Immunol 103 (2020) $126-134$.

[10] S.K. Nayak, Probiotics and immunity: a fish perspective, Fish Shellfish Immunol 29(1) (2010) 2-14.

[11] S. Viaud, F. Saccheri, G. Mignot, T. Yamazaki, R. Daillère, D. Hannani, D.P. Enot, C. Pfirschke, C. Engblom, M.J. Pittet, A. Schlitzer, F. Ginhoux, L. Apetoh, E. Chachaty, P.L. Woerther, G. Eberl, M. Bérard, C. Ecobichon, D. Clermont, C. Bizet, V. Gaboriau-Routhiau, N. Cerf-Bensussan, P. Opolon, N. Yessaad, E. Vivier, B. Ryffel, C.O. Elson, J. Doré, G. Kroemer, P. Lepage, I.G. Boneca, F. Ghiringhelli, L. Zitvogel, The intestinal microbiota modulates the anticancer immune effects of cyclophosphamide, Science 342(6161) (2013) 971-6.

[12] W.Z. Stephens, A.R. Burns, K. Stagaman, S. Wong, J.F. Rawls, K. Guillemin, B.J. Bohannan, The composition of the zebrafish intestinal microbial community varies across development, Isme j 10(3) (2016) 644-54.

[13] T. Rőszer, The invertebrate midintestinal gland ("hepatopancreas") is an evolutionary forerunner in the integration of immunity and metabolism, Cell Tissue Res 358(3) (2014) 685-95.

[14] K.F. Clark, A.R. Acorn, S.J. Greenwood, A transcriptomic analysis of American lobster (Homarus americanus) immune response during infection with the bumper car parasite Anophryoides haemophila, Dev Comp Immunol 40(2) (2013) 112-22.

[15] L.S. Dai, M.N. Abbas, S. Kausar, Y. Zhou, Transcriptome analysis of hepatopancraes of Procambarus clarkii challenged with polyriboinosinic polyribocytidylic acid (poly I:C), Fish Shellfish Immunol 71 (2017) 144-150.

[16] Z. Du, Y. Jin, D. Ren, In-depth comparative transcriptome analysis of intestines of red swamp crayfish, Procambarus clarkii, infected with WSSV, Sci Rep 6 (2016) 26780.

[17] J. Qin, Y. Li, Z. Cai, S. Li, J. Zhu, F. Zhang, S. Liang, W. Zhang, Y. Guan, D. Shen, Y. Peng, D. Zhang, Z. Jie, W. Wu, Y. Qin, W. Xue, J. Li, L. Han, D. Lu, P. Wu, Y. Dai, X. Sun, Z. Li, A. Tang, S. Zhong, X. Li, W. Chen, R. Xu, M. Wang, Q. Feng, M. Gong, J. Yu, Y. Zhang, M. Zhang, T. Hansen, G. Sanchez, J. Raes, G. Falony, S. Okuda, M. Almeida, E. LeChatelier, P. Renault, N. Pons, J.M. Batto, Z. Zhang, H. Chen, R. Yang, W. Zheng, S. Li, H. Yang, J. Wang, S.D. Ehrlich, R. Nielsen, O. Pedersen, K. Kristiansen, J. Wang, A metagenome-wide association study of gut microbiota in type 2 diabetes, Nature 490(7418) (2012) 55-60. 
[18] M.G. Grabherr, B.J. Haas, M. Yassour, J.Z. Levin, D.A. Thompson, I. Amit, X. Adiconis, L. Fan, R. Raychowdhury, Q. Zeng, Z. Chen, E. Mauceli, N. Hacohen, A. Gnirke, N. Rhind, F. di Palma, B.W. Birren, C. Nusbaum, K. Lindblad-Toh, N. Friedman, A. Regev, Full-length transcriptome assembly from RNA-Seq data without a reference genome, Nat Biotechnol 29(7) (2011) 644-52.

[19] M.J. Hubler, A.J. Kennedy, Role of lipids in the metabolism and activation of immune cells, J Nutr Biochem 34 (2016) 1-7.

[20] N.J. Pillon, P.M. Azizi, Y.E. Li, J. Liu, C. Wang, K.L. Chan, K.E. Hopperton, R.P. Bazinet, B. Heit, P.J. Bilan, W.L. Lee, A. Klip, Palmitate-induced inflammatory pathways in human adipose microvascular endothelial cells promote monocyte adhesion and impair insulin transcytosis, Am J Physiol Endocrinol Metab 309(1) (2015) E35-44.

[21] 0. Adam, Dietary fatty acids and immune reactions in synovial tissue, Eur J Med Res 8(8) (2003) 381-7.

[22] C.C. DiRusso, H. Li, D. Darwis, P.A. Watkins, J. Berger, P.N. Black, Comparative biochemical studies of the murine fatty acid transport proteins (FATP) expressed in yeast, J Biol Chem 280(17) (2005) 16829-37.

[23] C. López-Otín, M.A. Blasco, L. Partridge, M. Serrano, G. Kroemer, The hallmarks of aging, Cell 153(6) (2013) 1194-217.

[24] L. Papa, G. Manfredi, D. Germain, SOD1, an unexpected novel target for cancer therapy, Genes Cancer 5(1-2) (2014) 15-21.

[25] C.J. Banks, J.L. Andersen, Mechanisms of SOD1 regulation by post-translational modifications, Redox Biol 26 (2019) 101270.

[26] J.C. Espín, R. Varón, L.G. Fenoll, M.A. Gilabert, P.A. García-Ruíz, J. Tudela, F. García-Cánovas, Kinetic characterization of the substrate specificity and mechanism of mushroom tyrosinase, Eur J Biochem 267(5) (2000) 1270-9.

[27] G.E. Kaiko, T.S. Stappenbeck, Host-microbe interactions shaping the gastrointestinal environment, Trends in Immunology 35(11) (2014) 538-548.

[28] L.V. Hooper, A.J. Macpherson, Immune adaptations that maintain homeostasis with the intestinal microbiota, Nat Rev Immunol 10(3) (2010) 159-69.

[29] W.J. Lee, K. Hase, Gut microbiota-generated metabolites in animal health and disease, Nat Chem Biol 10(6) (2014) 416-24.

[30] J. Xiong, J. Zhu, W. Dai, C. Dong, Q. Qiu, C. Li, Integrating gut microbiota immaturity and disease-discriminatory taxa to diagnose the initiation and severity of shrimp disease, Environ Microbiol 19(4) (2017) 1490-1501.

[31] E. Garibay-Valdez, M. Martínez-Porchas, K. Calderón, F. Vargas-Albores, L. Martínez-Córdova, Taxonomic and functional changes in the microbiota of the white shrimp (Litopenaeus vannamei) associated with postlarval ontogenetic development, Aquaculture 518 (2019) 734842.

[32] N.R. Shin, T.W. Whon, J.W. Bae, Proteobacteria: microbial signature of dysbiosis in gut microbiota, Trends Biotechnol 33(9) (2015) 496-503.

[33] A.J. Redford, N. Fierer, Bacterial succession on the leaf surface: a novel system for studying successional dynamics, Microb Ecol 58(1) (2009) 189-98.

[34] H. Teeling, B.M. Fuchs, D. Becher, C. Klockow, A. Gardebrecht, C.M. Bennke, M. Kassabgy, S. Huang, A.J. Mann, J. Waldmann, M. Weber, A. Klindworth, A. Otto, J. Lange, J. Bernhardt, C. Reinsch, M. Hecker, J. Peplies, F.D. Bockelmann, U. Callies, G. Gerdts, A. Wichels, K.H. Wiltshire, F.O. Glöckner, T. Schweder, R. Amann, Substrate-controlled succession of marine bacterioplankton populations induced by a phytoplankton bloom, Science 336(6081) (2012) 608-11.

[35] F. Cornejo-Granados, A.A. Lopez-Zavala, L. Gallardo-Becerra, A. Mendoza-Vargas, F. Sánchez, R. Vichido, L.G. Brieba, M.T. Viana, R.R. Sotelo-Mundo, A. Ochoa-Leyva, Microbiome of Pacific Whiteleg shrimp reveals differential bacterial community composition between Wild, Aquacultured and AHPND/EMS outbreak conditions, Sci Rep 7(1) (2017) 11783.

[36] S. Gao, L. Pan, F. Huang, M. Song, C. Tian, M. Zhang, Metagenomic insights into the structure and function of intestinal microbiota of the farmed Pacific white shrimp (Litopenaeus vannamei), Aquaculture 499 (2019) 109-118.

[37] Y.J. Chou, J.H. Chou, M.C. Lin, A.B. Arun, C.C. Young, W.M. Chen, Vogesella perlucida sp. nov., a non-pigmented bacterium isolated from spring water, Int J Syst Evol Microbiol 58(Pt 12) (2008) 2677-81.

[38] N.O.G. Jørgensen, K.K. Brandt, O. Nybroe, M. Hansen, Vogesella mureinivorans sp. nov., a peptidoglycan-degrading bacterium from lake water, Int J Syst Evol Microbiol 60(Pt 10) (2010) 2467-2472.

[39] M. Hjelm, A. Riaza, F. Formoso, J. Melchiorsen, L. Gram, Seasonal Incidence of Autochthonous Antagonistic Roseobacter spp. and Vibrionaceae Strains in a Turbot Larva (Scophthalmus maximus) Rearing System, Applied \& Environmental Microbiology 70(12) (2004) 7288-7294.

[40] S.G. Joshi, G.M. Litake, Acinetobacter: An emerging pathogenic threat to public health, World Journal of Clinical Infectious Diseases 3(3) (2013) 25-36.

[41] E. Blacher, M. Levy, E. Tatirovsky, E. Elinav, Microbiome-Modulated Metabolites at the Interface of Host Immunity, J Immunol 198(2) (2017) 572-580.

\section{Tables}

Table 1 Specimen sequencing data

Page $9 / 16$ 


\begin{tabular}{|llllllll|}
\hline sample & Raw reads & Clean reads & Clean bases & Error rate & Q20 & Q30 & GC pct \\
\hline SI1 & 49519914 & 48843676 & $7.33 G$ & 0.03 & 97.83 & 93.8 & 43.68 \\
SI2 & 53486822 & 52693916 & $7.9 \mathrm{G}$ & 0.03 & 97.8 & 93.9 & 48.04 \\
\hline SI3 & 40823606 & 40342820 & $6.05 G$ & 0.03 & 97.77 & 93.8 & 47.69 \\
\hline LI1 & 52037658 & 51304474 & $7.7 G$ & 0.03 & 97.88 & 94 & 47.57 \\
LI2 & 52785386 & 51688968 & $7.75 G$ & 0.03 & 97.76 & 93.6 & 48.71 \\
\hline LI3 & 56999874 & 55817174 & $8.37 G$ & 0.03 & 97.7 & 93.5 & 48.41 \\
\hline
\end{tabular}

Table 2 Spliced transcripts and gene sequence length

\begin{tabular}{|llllllll|}
\hline & Min length & Mean length & Median length & Max length & N50 & N90 & Total nucleotides \\
\hline Transcripts & 301 & 1589 & 792 & 32544 & 2999 & 579 & 135061303 \\
Genes & 301 & 1367 & 701 & 32544 & 2468 & 511 & 60637958 \\
\hline
\end{tabular}

Table 3 Gene annotation statistics

\begin{tabular}{|lll|}
\hline Database & Number.of.Unigenes & Percentage \\
\hline Annotated in NR & 14505 & 32.68 \\
\hline Annotated in NT & 5677 & 12.79 \\
\hline Annotated in KO & 5684 & 12.8 \\
\hline Annotated in SwissProt & 10306 & 23.22 \\
\hline Annotated in PFAM & 14373 & 32.39 \\
\hline Annotated in GO & 14373 & 32.39 \\
\hline Annotated in KOG & 5889 & 13.27 \\
\hline Annotated in all Databases & 1642 & 3.7 \\
\hline Annotated in at least one Database & 20369 & 45.9 \\
\hline Total Unigenes & 44372 & 100 \\
\hline
\end{tabular}

Table 3 Enrichment and annotation of the top ten species of intestinal flora at the phylum level.

\begin{tabular}{|c|c|c|c|c|c|c|c|c|c|}
\hline Taxonomy & Proteobacteria & $\begin{array}{l}\text { Unidentified } \\
\text { Bacteria }\end{array}$ & Firmicutes & Tenericutes & Chloroflexi & Actinobacteria & Acidobacteria & Bacteroidetes & Rokubacteria \\
\hline SI & 0.395748 & 0.009636 & 0.058275 & 0.002842 & 0.053386 & 0.053527 & 0.046779 & 0.051246 & 0.022337 \\
\hline LI & 0.492159 & 0.162837 & 0.016653 & 0.046592 & 0.009403 & 0.031084 & 0.01318 & 0.029681 & 0.004011 \\
\hline
\end{tabular}

\section{Figures}




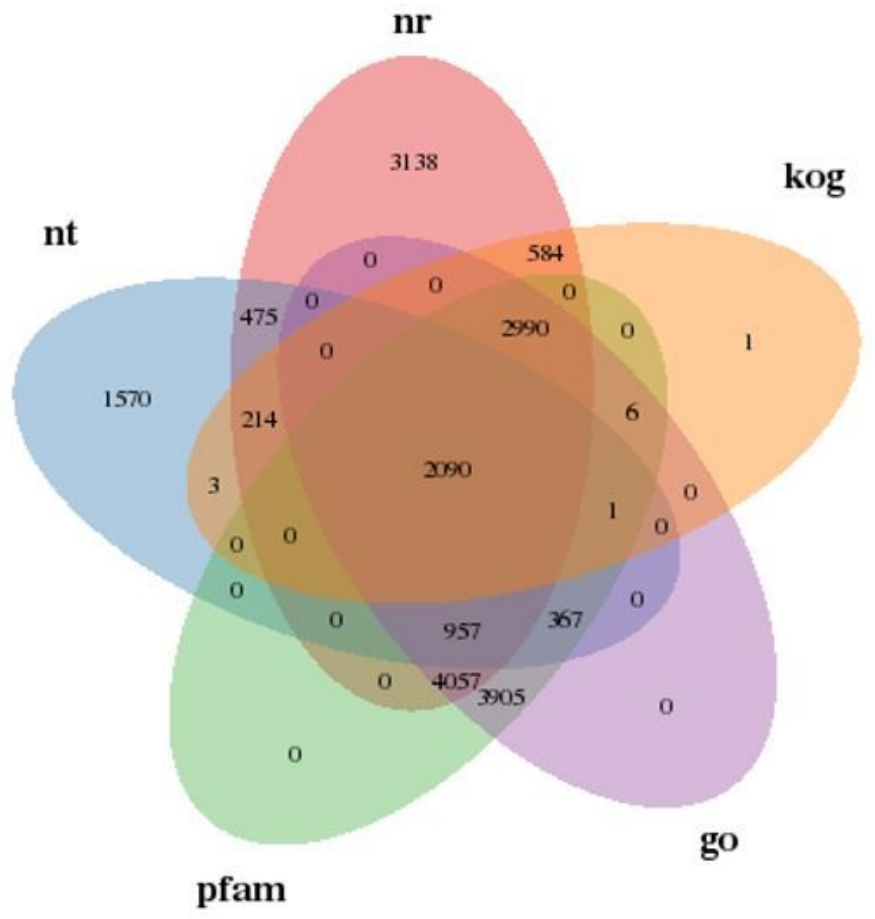

Figure 1

Venn diagram showing overlaps among NR, NT, KOG, GO and Pfam. Numbers in each circle indicate the number of annotated unigenes.

A

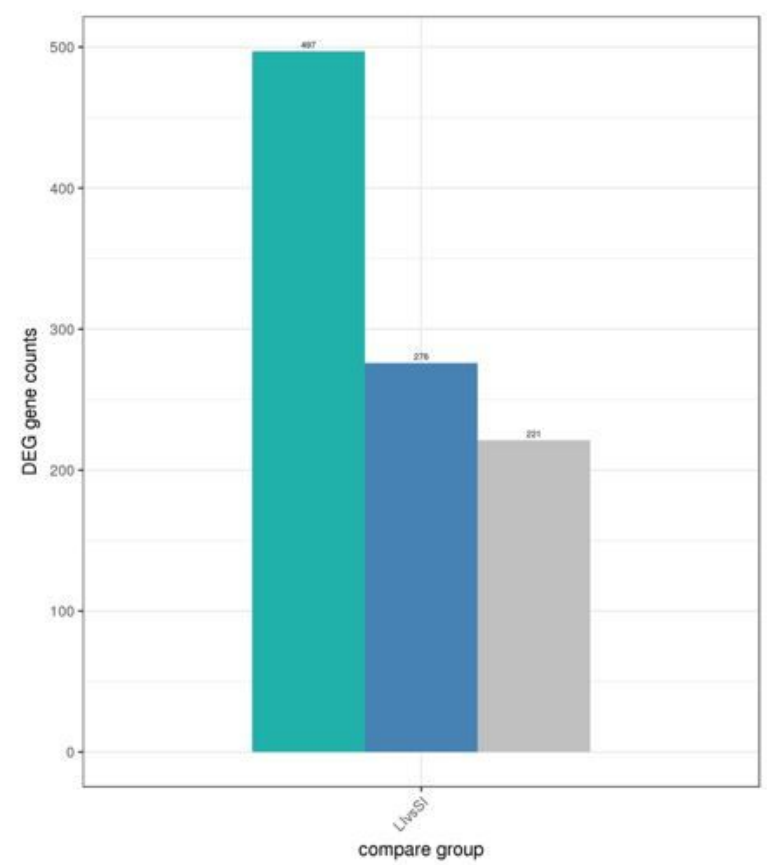

B

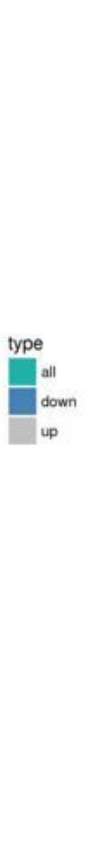

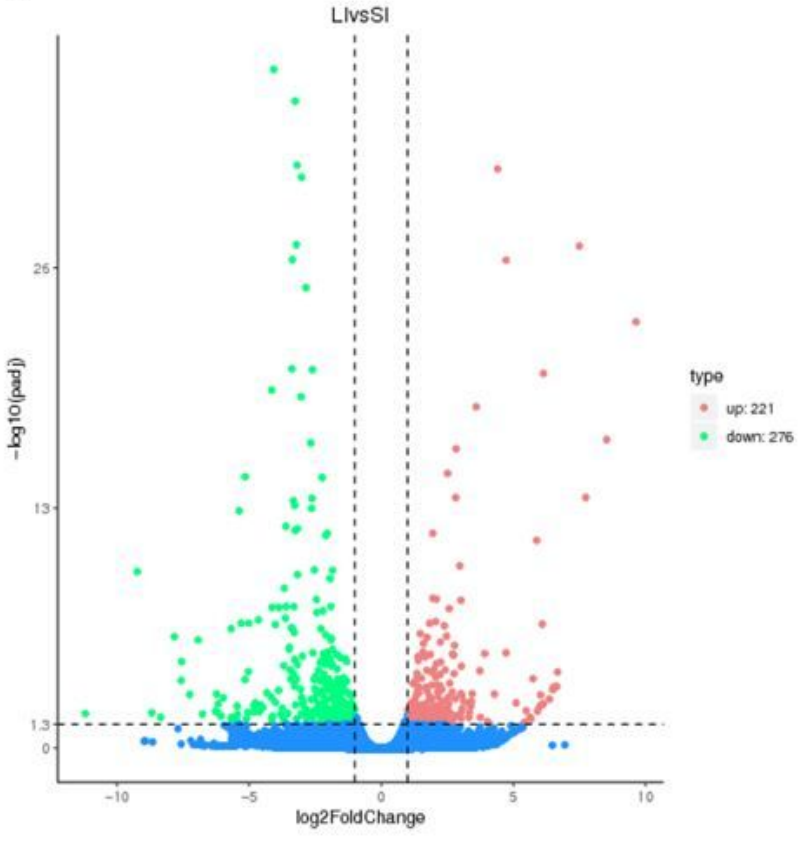

Figure 2

Statistical histograms of the number of DEGs and volcano plots of DEGs. 


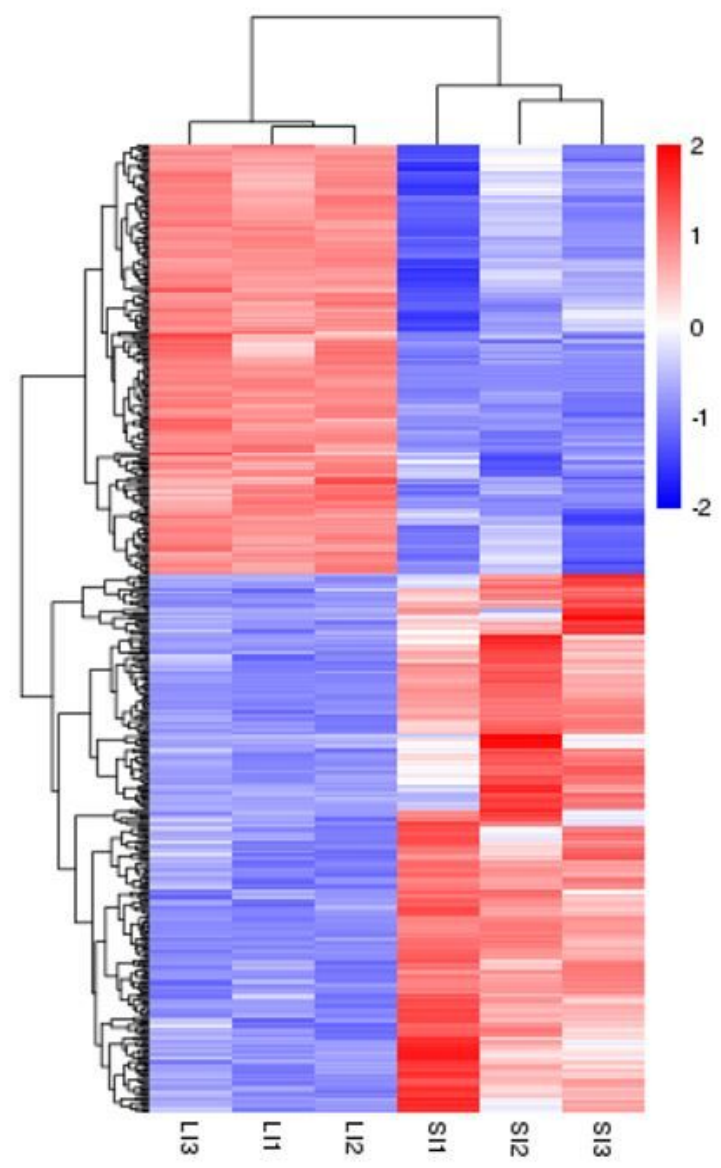

Figure 3

Differential gene clustering heat map. Columns in different graphs represent different samples, while different rows represent different genes. 


\section{KEGG Classification}

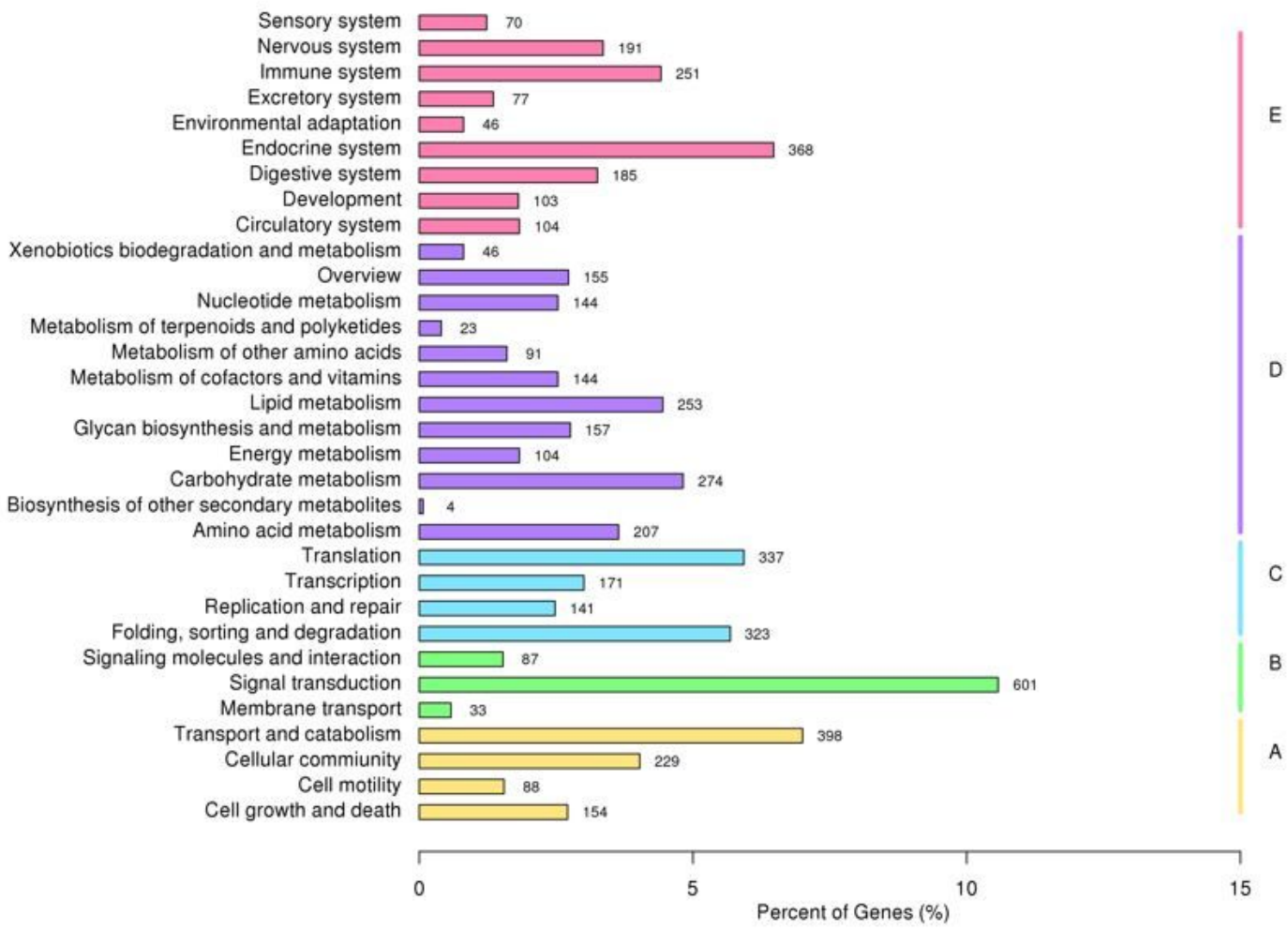

Figure 4

KEGG metabolic pathway classification statistics. The ordinate is the name of the KEGG metabolic pathway, while the abscissa is the number of genes annotated for the pathway and their proportion to the total number of annotated genes. Based on the KEGG metabolic pathways involved, the genes are classified into five branches: a) cellular processes, b) environmental information processing, c) genetic information processing, d) metabolism and e) organic systems. 


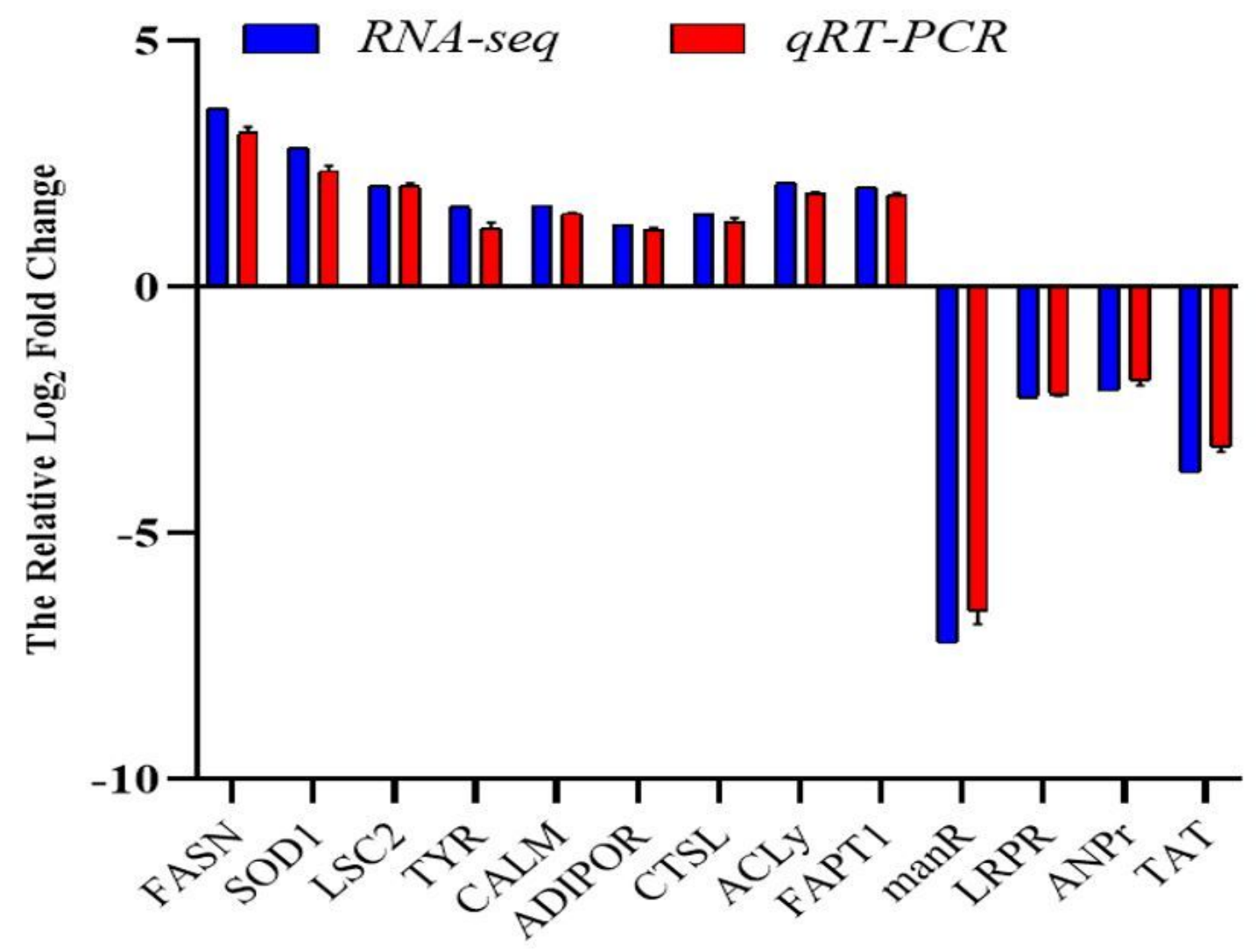

Figure 5

Expression levels of candidate unigenes were assessed by RT-qPCR, and the expression trend was the same as that of RNA-Seq.
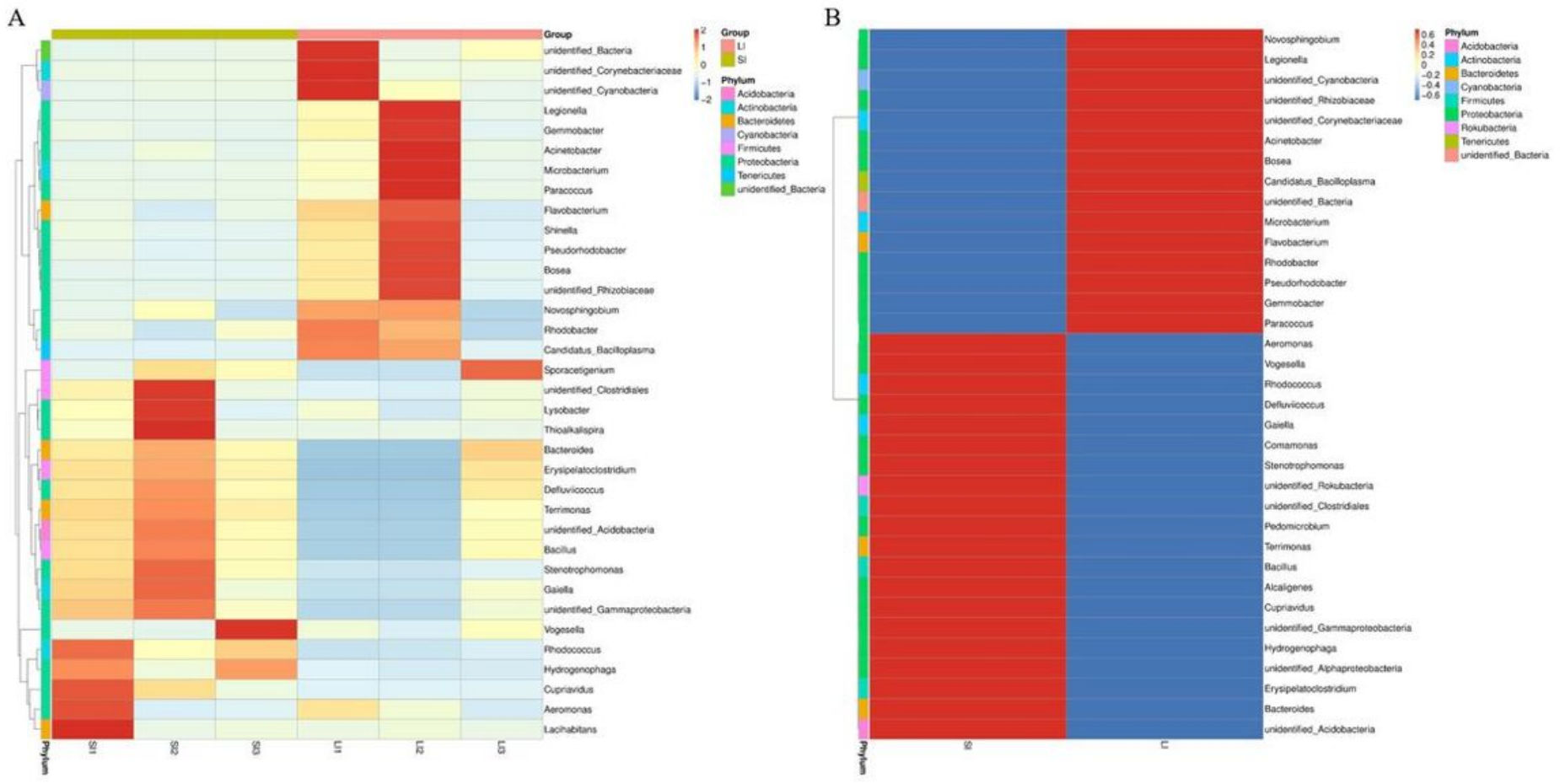

Figure 6

Species abundance clustering map 


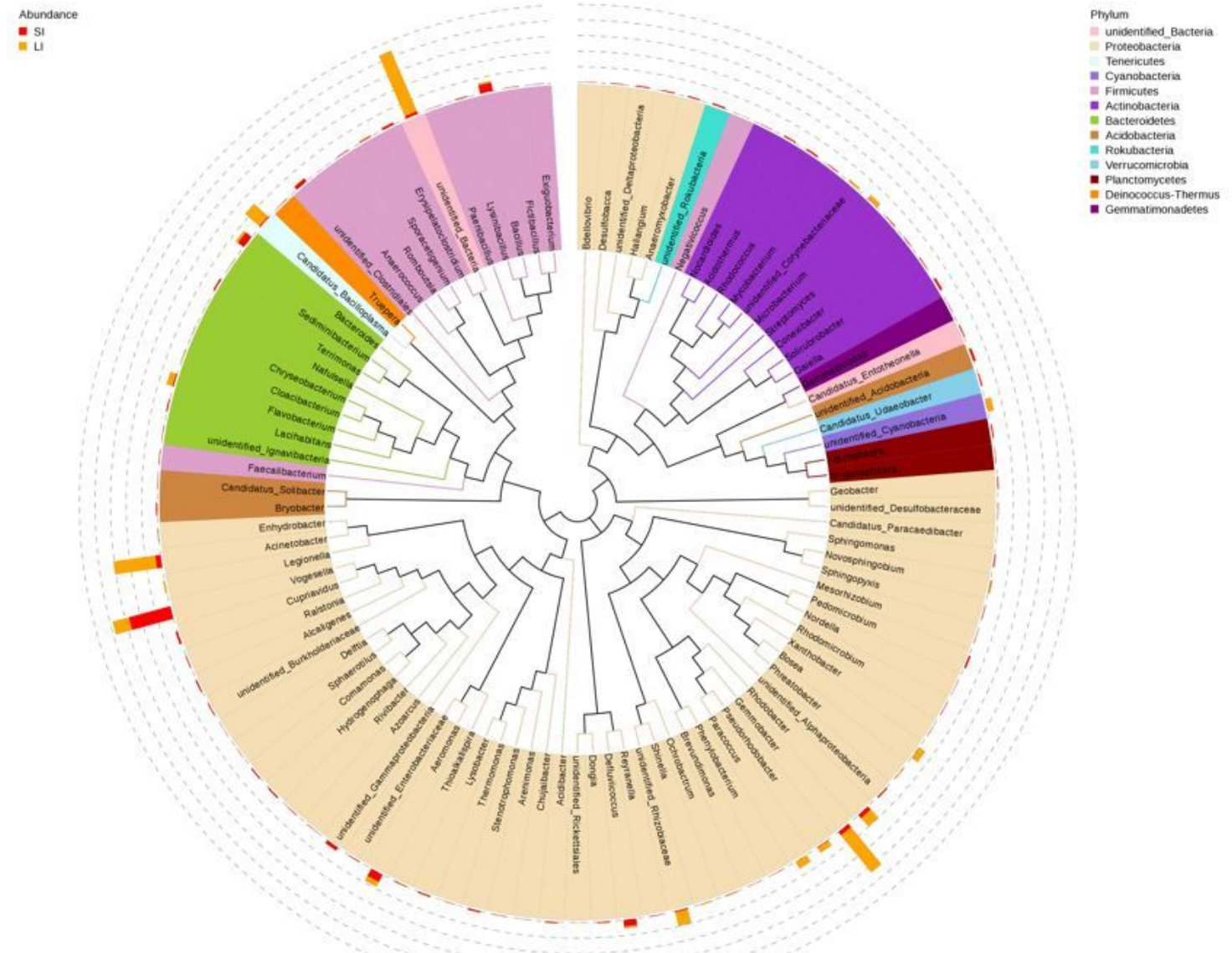

Figure 7

Genus-level species phylogeny

A

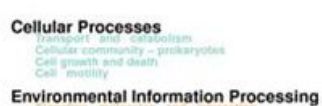

Environmental Information Processing

Genetic Information Processing

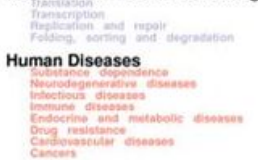

Metabolism

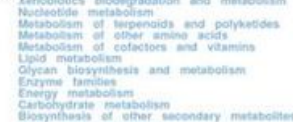

Organismal Systems

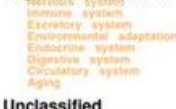

Unclassified

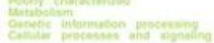

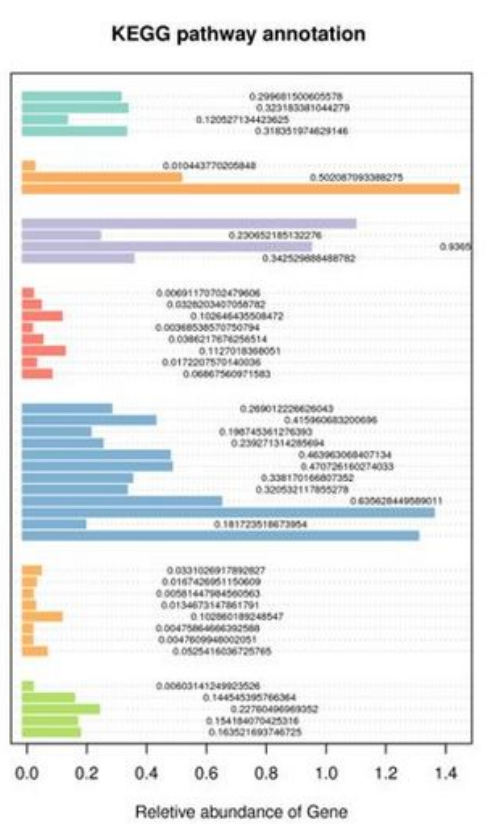

B

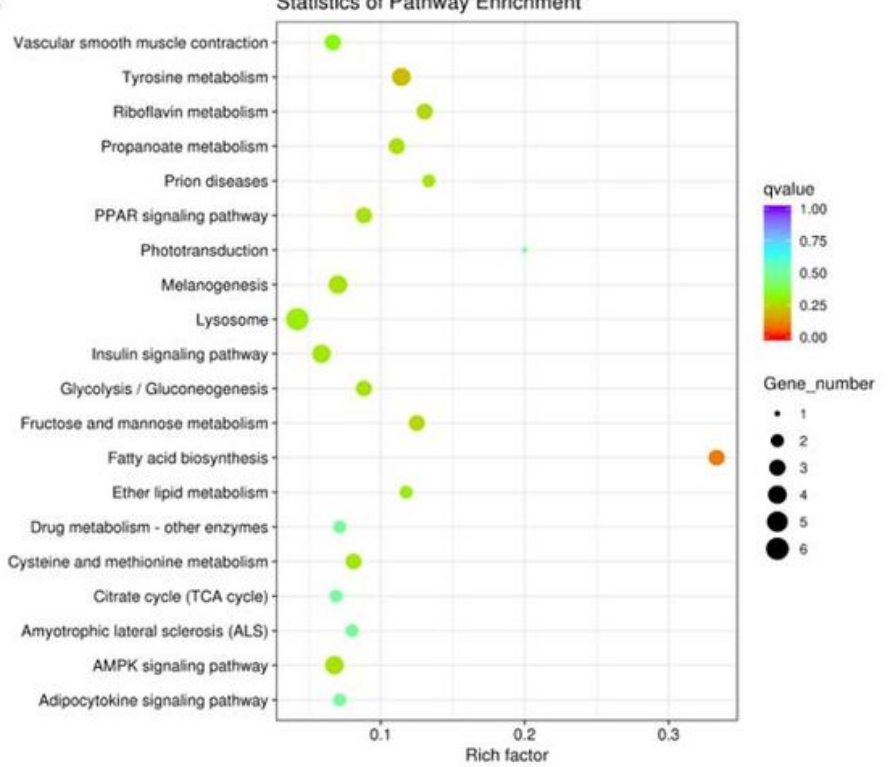

Figure 8 
Intestinal microbial and transcriptomic KEGG pathway enrichment scatter plots. A. Intestinal microbes KEGG genes. B. Transcriptomic KEGG pass-through enrichment scatter plot.
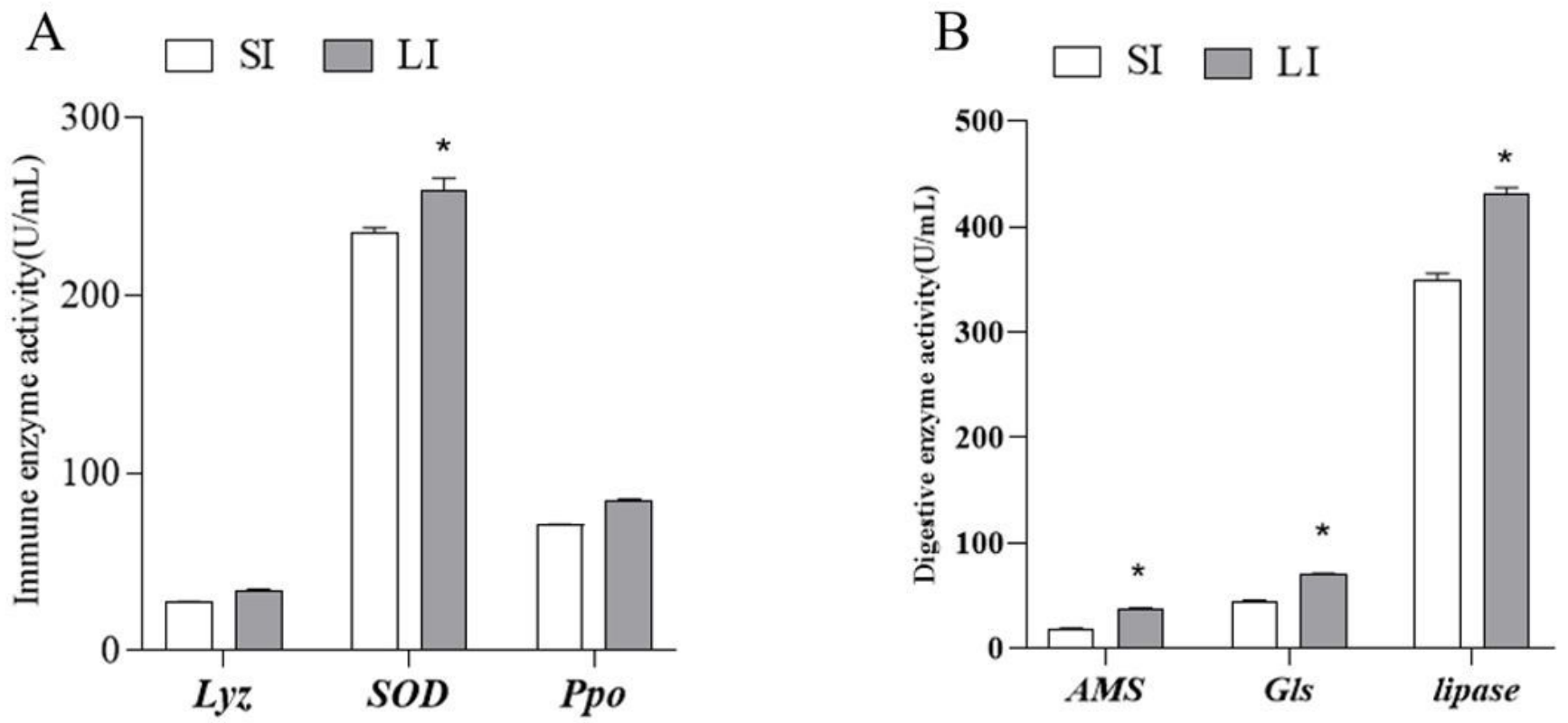

Figure 9

Humoral factor viability test A): Immune enzyme viability test; B): Digestive enzyme viability test.

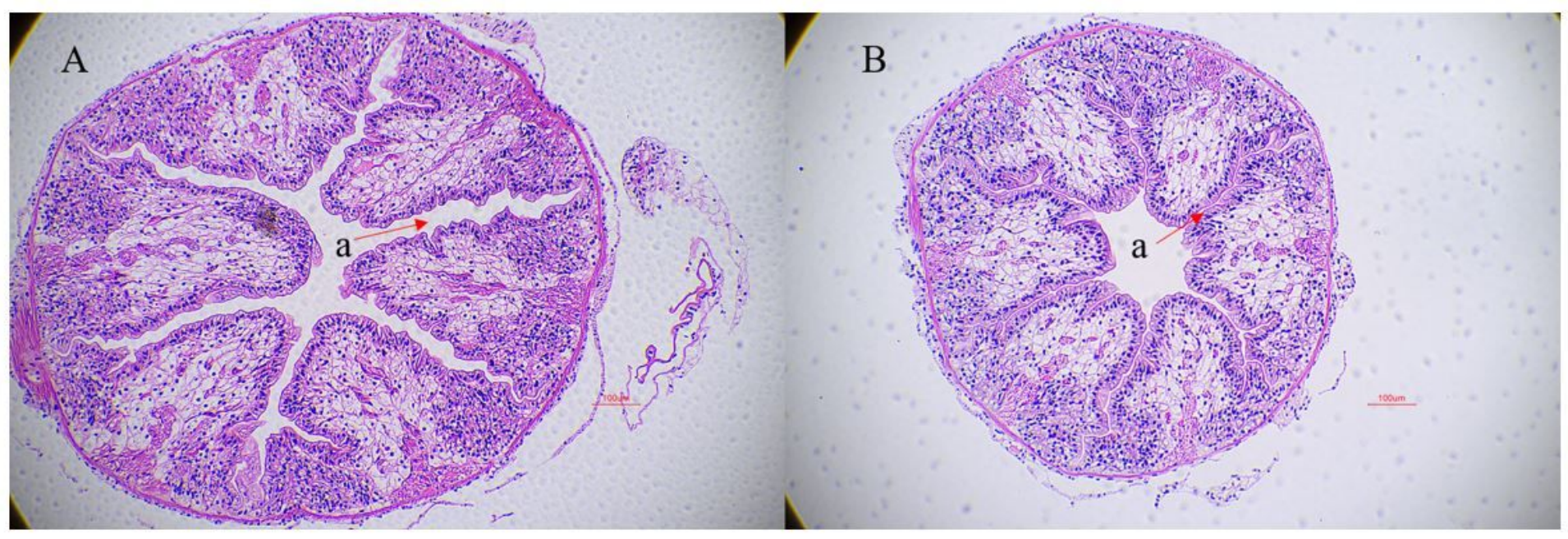

Figure 10

Histological sections of the intestine from different groups 200X. A): LI group Transverse section of the intestines; B): SI group Transverse section of the intestines.

\section{Supplementary Files}

This is a list of supplementary files associated with this preprint. Click to download.

- TableS1.docx

- S1.jpg

- S2.jpg 